\title{
Signs of Appropriation: A Semiotic Account of Breakdowns with IoT Technology
}

\author{
Bruno A. Chagas \\ Department of Informatics \\ PUC-Rio \\ Rio de Janeiro, Brazil \\ bchagas@inf.puc-rio.br
}

\author{
David F. Redmiles \\ Department of Informatics \\ University of California, Irvine \\ Irvine, USA \\ redmiles@ics.uci.edu
}

\author{
Clarisse S. de Souza \\ Department of Informatics \\ PUC-Rio \\ Rio de Janeiro, Brazil \\ clarisse@inf.puc-rio.br
}

\begin{abstract}
The Internet of Things (IoT) is an emerging technology powered by smart devices that increasingly populate our environment and transform our practices. Understanding how technologies are appropriated is a key asset for designing them successfully. In this paper, we present findings from an insitu study where we observed how people use and adapt commercially available smart home technology, in real practice, and over time ${ }^{1}$. By using semiotic theory and thematic analysis to investigate data from diaries, questionnaires and interviews collected from a group of eleven participants, we characterized IoT appropriation as interpretive states that can evolve overtime. We also identified eight classes of appropriation breakdowns and derived two semiotic qualities for IoT technologies. We propose that, together, they have the potential to support productive decision making by IoT designers, as well as to raise new research questions on the topic.
\end{abstract}

Keywords-internet of things, appropriation, semiotic engineering, end-user development, in situ study

\section{INTRODUCTION}

The Internet of Things (IoT) is now widely known and marketed as one of the main technological advancements of our time. It is an emerging technology powered by smart devices that are likely to pervade our world and practices more and more in the near future. These devices introduce new capabilities of sensing and acting, as well as a layer of connectivity and computational power over our everyday objects and environments, allowing for new degrees of responsiveness and novel modes of interaction. For example, fitness and wellness devices already sense our bodies' signals and help to evaluate health conditions and adjust our activities accordingly; smart homes sense current weather conditions and can adjust the internal temperature to meet their inhabitants' preferences; smart cities in the future should be able to optimize traffic and resources consumption by being able to react to current urban conditions in real time. Despite the great potential in several areas and applications, technology adoption is not instantaneous, it takes time and is influenced by several factors, both at the individual [2] and social [3] levels. The process of technology adoption by people over time is called appropriation and comprises the adaptation of technology to their pre-existing practices and vice-versa.

A critical moment during the appropriation process is when a breakdown occurs. In communication, breakdowns refer to disruptions and misunderstandings in the natural flow of a conversation [4], [5]. A communication breakdown demands interlocutors' attention and action in order to repair the conversation and restore mutual understanding, making their focus shift from the natural flow of conversation to a repair process [6]. Similarly, in human-computer interaction, a breakdown refers to those moments when technology demands the focus of user's attention, which usually happens

Research partially funded by two Brazilian government agencies, CAPES (Grant No. 88887-122734/2016-00) and CNPq (Grants No. 153902/2015-9, 304224/2017-0, and 140568/2018-2). upon a failure [7]. In such situations, users are forced to make sense of what is happening and they will mentally break down (i.e. deconstruct) the technology, its properties and their own understanding about it. Breakdowns are critical for appropriation because they correspond to those situations when users get motivated, urged, or at least intrigued, to learn and adapt the technology in order to repair interaction, that is, their "conversation with the technology."

We can imagine that users face old and new breakdowns when interacting with IoT technologies. A device not responding due to a connection problem is probably a kind of situation we have all faced at least once in our lives. On the other hand, a smart home that controls lights automatically may generate breakdowns more closely associated with the novelties introduced by the IoT. For example, suppose your smart home turns all lights off every night at 10 p.m. and, on some particular occasion, you need them to stay on after that time (because of a party, a repair you need to make in your house, or an article you need to finish). When the lights go off in such situation, you will disagree with the system and interact with it in order to restore what is reasonable to you at that particular time. Thus, breakdowns are not necessarily associated with (technical) failures (only). Breakdowns occur whenever there is a mismatch between users' expectations and the technology, which often happens because of users' evolving or changing expectations, a natural part of the appropriation process.

In research about IoT, appropriation is a rather new topic. Researchers and designers need to understand how appropriation takes place in order to be able to provide strong support for it when designing interaction with IoT technology. Assuming that the breakdowns people have when interacting with IoT technology are critical moments for the appropriation process, we conducted a diary study with 11 novice users using a set of 5 commercially available smart home devices over the course of four weeks in their natural settings. We took Semiotic Engineering as a theoretical lens to analyze the data and understand the breakdowns we observed throughout the study, relating them to the appropriation of the technology by the users. Our contribution is a semiotic account of appropriation and two derived qualities that can help designers to address appropriation support in IoT technologies, namely cross-interface consistency and cross-device coherence ${ }^{1}$.

This paper is organized in seven sections, starting with the present introduction. In Section II, we present some background regarding the topics approached in this work, namely IoT, appropriation and Semiotic Engineering. In Section III, we describe the empirical study we conducted along with our data analysis approach. In Section IV, we describe our main results and findings organized according to our data analysis approach. In Section V, we present and discuss our contributions in face of our main findings. In

${ }^{1}$ A shorter version of this paper has been presented in the $17^{\text {th }}$ Brazilian Symposium on Human Factors in Computing Systems (IHC 2018) with the title "Observed Appropriation of IoT Technology: A Semiotic Account" [1]. 
Section VI, we discuss some of the limitations of this work and point to some directions for future work. Finally, we conclude the paper in Section VII.

\section{BACKGROUND}

Three major areas come together to form the basis for our research. Our domain is the Internet of Things (IoT). Our theoretic lens is Semiotic Engineering. The problem we studied is closely related to that of technology appropriation.

\section{A. Internet of Things (IoT)}

The IoT has gained increasing attention from research, governments and companies around the world because of its potential social and economic impact. The concept is largely popularized now. Despite its original, somewhat restricted historical meaning related to the use of RIFD tags in industry [8], nowadays the IoT is largely studied with a much broader meaning in engineering [9]-[15] and as the infrastructure for specific applications such as smart homes [16], smart cities [17], industry [18], healthcare [19], and many more. Despite the several visions, approaches and focuses in IoT research, in general they share the core idea of having everyday objectsthings - endowed with computational power and network connectivity, usually also with the Internet and wirelessly. Embedded miniaturized computing technologies allow objects to become "smart," in the sense that they are enhanced, or more responsive than their "dumb" counterparts.

A smart device is a general term used in the IoT domain to refer to this kind of object. They are capable of sensing and actuating in the physical world and have different degrees of sophistication. At the current stage of IoT technology, smart devices such as smart thermostats, smart lights, smart sensors, smart voice assistants, etc. can be found at regular electronic retail stores in several countries. Smart devices comprise the building blocks of the IoT [20]. They embody different parts of the grand IoT vision and their specialized functions can be combined with each other in order to produce more complex behaviors. Users are left in charge of installing and configuring them in actual contexts of use. More importantly, users should figure out how to effectively use smart devices, incorporating them into their everyday practices, adapting devices to practices and practices to devices. In other words, users will appropriate IoT smart devices.

\section{B. Appropriation}

Appropriation has been defined as "the way in which technologies are adopted, adapted and incorporated into working practices" [21] and "the process of taking something that belongs to others and making it one's own" [22]. As the word's roots suggest, it has a double meaning of "making it appropriate" (suitable) to somebody or some group in their situated contexts, tasks and practices, and "taking something for one's own possession and use."

The concept is often used in Education research and practice, although often in an informal way and without a precise definition or account. When a learner is observed applying a skill or knowledge wisely he or she is said to have appropriated it (e.g. [23], [24]). In this vein, Rogoff traces the first appearances of the term to (philosopher and semiotician) Bakhtin "who argued that the words people use belong partially to others, as they appropriate words from others and adapt them to their own purposes" [25]. We have not been studying Bakhtin deeply in the context of this research, but his dialogic philosophy of language points to an interesting direction of theoretical research linking communication (language in use) and appropriation in Education e.g. [26].

Appropriation of technology appears in Human-Computer Interaction (HCI) literature usually in reference to the process by which users become able to make effective use of technology in their personal contexts, fitting and incorporating systems into their actual practices, often in creative ways not originally anticipated by designers [27]. It is closely related to the idea of customization but it is a broader term that emphasizes the "ongoing, incremental adaptation of interactive technologies [...] inherent to the emergence of practice" [21]-rather than the isolated act or task of customizing a system alone.

Appropriation has been investigated with different kinds of systems and in different domains, such as collaborative groupware software [21], [28], open software ecosystems [29], and mobile technologies [30]-[32]. However, appropriation of IoT technology has not been extensively investigated yet. With IoT technology gaining popularity, researchers are starting to address this gap [33], [34] but more research is needed. Our work's primary goal is to address this gap.

Appropriation unfolds in both the individual and the social levels (that is, regarding technology adoption and adaptation by groups, communities of practice or the whole society). Gaskin \& Lyytinen say that research about technology appropriation has privileged the latter in detriment of the former [2] (their own study being a counterbalance to this vision). Indeed, we can observe that the majority of the papers cited in the previous paragraph take a socio-technical perspective over the topic. Both aspects are probably intertwined and our approach starts with the primary interest in appropriation at the individual level.

Carroll proposed that appropriation is actually part of the design process, since technology in use becomes different from technology as designed [35]. Design and appropriation form a continuous cycle of design for appropriation, appropriation by users and design from appropriation, when technology evolves based on what designers learn from users. More specific guidelines have been proposed to orient the design for appropriation [27]. Instruments to enable and support designers to learn from and negotiate with their users support the design from appropriation and have also been proposed [36]. These works address the relationship between appropriation and design as an effort to improve the support for appropriation in technology.

Carroll has contributed to theory by proposing a conceptual model to describe appropriation at a macro level, identifying factors that repel or attract users to appropriate or not technology as designed and criteria that reinforce the appropriation process or cause disappropriation of technology by young people [37]. Karapanos et al. related appropriation to the larger process of how user experience develops over time. Studying iPhone adoption by new users, they identified four stages of what they called "temporality of experience:" anticipation, orientation, incorporation, and identification, appropriation occurring mainly during the orientation and incorporation phases and being influenced by forces such as increasing familiarity and functional dependency [38]. Similarly, Bødker \& Christiansen proposed four stages of the appropriation process of "the world of apps": anticipation, 
initial familiarity, development of repertoires of routines, and the development of new forms of use [22].

Most of these accounts were primarily derived from observations and empirical data of users of a particular system. A challenge with these approaches is to make "appropriate" sense and use of results derived from different systems, used by different people, in different settings and obtained through different methods. One way to overcome these difficulties is to turn to theories as a set of principles and knowledge that can explain observed facts at a deeper level. A theory can help reveal important connections and relationships hidden underneath the surface of observed phenomena by guiding research questions and analysis in certain directions. In our case, we chose Semiotic Engineering as the theory to guide our analysis in order to complement previous knowledge about the topic in other dimensions.

\section{Semiotic Engineering}

Semiotic Engineering [39] is a theory of HCI that views interaction between users and computer technologies as a special case of computer-mediated human communication. According to this theory, any system communicates a message from its designers to its users, which expresses the designer's view about who the users are and what they want or need to do with the system. This message is encoded and communicated by the system's interface signs (hence the name, semiotic), and unfolds to the users when they interact with the system, at use time. The software is thus a deputy (or proxy) of the system's designers, in charge of telling users what the designers mean. Over time, interaction (that is, users communicating with the system interface, the designer's deputy) reveals the underlying designers' message about the software. Interaction is thus a metacommunication process: a system-user communication about the designer-user communication that happens through the software interface.

By looking at interaction as communication, we bring to the foreground of interaction (as the phenomenon under investigation and object of study) the fact that every single aspect of interfaces is both designed, from the designer's perspective, and interpretive, from the users' perspective. A system's interface is an artifact intentionally designed by somebody with a set of purposes and assumptions in mind. During use, the interface will be interpreted by different people with different backgrounds and different contexts, whose interpretation may or may not be in line with the intended original design. The interpretation process undertaken by users is very important to Semiotic Engineering. According to semiotic theory [40], a sign has no "natural," objective or pre-defined meaning. To put it in simple proverbial terms, "meaning is in the mind of the beholder." Moreover, every new encounter with a sign has the potential to trigger further interpretations. Therefore, the same sign is actually interpreted in different ways (even if slightly) by different people and even by the same person, at different times. The underlying interpretive process is a complex one.

To illustrate it, consider this example. While the first author worked on this paper in a hotel room with an unfamiliar coffee machine by his side, he sees three buttons on it with icons resembling a coffee cup, each one in a different color: red, blue and green. The buttons can be pushed but what they mean to him is probably different from what they mean to somebody who has actually read the user's manual or used the machine before. If we could ask them, the machine's designers would probably give us a rich explanation about each button.
The first author's immediate guess is that the buttons correspond to different cup sizes (small, medium and large). After pressing all three buttons, they seem to have produced the same outcome, dispensing just enough hot water to fill a small cup. Therefore, right now, they all have the same meaning to the interpreter: "Press to make coffee!" However, this meaning is not static or definitive. So, he is now wondering if they could have meant different coffee temperatures, like: "very hot," "medium hot," and "not so hot." Or maybe the machine is just not working properly and his first guess was actually right. We may never know and we can stop the generation of possible explanations here, for sake of time and space. However, nothing prevents him from coming up with new explanatory hypotheses, at will. In fact, he may well have to generate new ones in face of counterfactuals (e. g. verifying that the temperature of the drink is the same regardless of which button he presses). Indeed, this process can go on indefinitely. In semiotic terms, this process of building explanatory hypotheses in our minds to explain (interpret or make sense of) the world around us is called unlimited semiosis and is driven by abduction or abductive reasoning, also called hypothetical reasoning.

Abduction is the operation of adopting an explanatory hypothesis in inferencing processes [40]. When facing a surprising fact or sign, we will try to make sense of it by coming up with reasonable hypotheses that can, in the current circumstances and context, explain it. A hypothesis can be as reasonable as the person's knowledge (that is, any previous information one has, e.g. coffee machines have buttons to make coffee, usually one is enough) and imagination allows it to. However, there is no guarantee that it is true. It will only remain true until evidence is found that indicates otherwise, a new surprising fact or sign that triggers a reassessment of the entire "theory" developed by the person.

Notice the important role of the surprise as the actual trigger that starts cycles of abductive reasoning. They allow wrong hypothesis to be corrected and right ones to be improved or extended, "on the fly." We can call these surprising facts breakdowns, in the same sense as communication breakdowns, disruptions in the natural flow of communication that demand from us extra work in order to restore mutual understanding between communication parties [41]. The first level of extra work is the abductive generation of hypothesis to explain the breakdown in order to inform, enable and support any further action e.g. an attempt to repair the communication.

The importance of breakdowns to human-computer interaction has been pointed before [7] but its potential seems still under explored. It has been defined as "the moment when the user becomes conscious of the properties of the system and has to mentally break down or decompose his or her understanding of the system in order to rationalize the problem experienced" [42]. Sharples says that "breakdowns occur when the routine of work is interrupted, by straightforward technical failures, such as the loss of communication line, by social problems, such as interpersonal conflict, or by mismatches of expectation, when users become frustrated that the equipment does not fit with their needs or preconceptions" [43]. A similar sense is used by Pipek \& Wulf related to the idea of technology as infrastructures supporting users' practices and the emergence of breakdowns as actual or perceived failures [44]. 
To Semiotic Engineering, interaction failures are studied as communication breakdowns. Communication breakdowns that happen during interaction are signs of the quality of the system's interface. The main operational property of a system's interface in this perspective is called communicability, "the property of software that efficiently and effectively conveys to users its underlying design intent and interactive principles" [45]. In this sense, Semiotic Engineering has proposed methods to evaluate the communicability of software interfaces [46], and one specifically targeted at detecting communication breakdowns that happen during user's interaction [47]. We will describe it further below.

\section{Methods}

We conducted a 4-week diary study [48, Ch. 10] with novice users of smart devices during the fall of 2017. Our primary goals were to observe how people would use and adapt IoT technology and to collect data about the breakdowns they would face. Fourteen subjects were recruited from undergraduate courses in Computer Science and related fields of an American university. Selection criteria were that participants were at least 18 years old, registered as an undergraduate student, had a mobile phone and were interested in IoT. Eleven participants completed the study, 7 males, 4 females, ages 20.5 on average (19 minimum, 25 maximum). Participants were compensated with 30 USD in cash and the devices (approximately U\$D 200 in value) used in the study if they completed it.

The technology was chosen in order to produce a small but minimally rich IoT ecosystem. Different functionalities and brands were chosen in order to avoid any sort of bias from a particular manufacturer. Devices should not demand specialized equipment or professional installation and participants should need only their mobile phone and Wi-Fi internet to use the devices. Moreover, devices should be able to combine with each other in flexible ways. The IFTTT online service ("If This Then That" - https://ifttt.com) was chosen in order to allow devices' combinations. Each participant received a set of:

- An Amazon Echo Dot (voice assistant);

- A LIFX smart colored LED light;

- A WeMo Insight Switch (smart power plug with energy metering);

- A myDLink Wi-Fi Motion Sensor;

- A Flic button (a Bluetooth push button).

The study was divided into three parts. First, participants came to a kick-off workshop where we collected basic demographic information, distributed the devices, and gave a presentation with quick demos. In the following 4 weeks, participants submitted 3-5 diary entries per week remotely through an online form answering open questions about how they were using the devices and the changes they performed. During this phase, participants were emailed a weekly "task" that was intended to stimulate them to use the devices and to explore some functionalities and possibilities of the technology. All tasks were proposed according to the following general instruction communicated verbally to the participants: "Take the devices you got and browse for applets for them in IFTTT," where each week we suggested different IFTTT services to combine (e.g. devices alone and together, date and time service, location). After 4 weeks, participants were invited to a final individual 40-minute interview about their overall experience and about particular situations they reported.

We performed a thematic analysis [49] of participants' 130 diary submissions and 11 final interviews. Data was coded for recurring and emergent themes with particular attention given to looking for breakdown situations. Whenever users explicitly reported a surprising fact (to them), an issue, or a change in some configuration (presumably as a consequence or an attempt to address a previous issue that they have had), a potential breakdown situation was marked. Around 430 breakdowns were initially identified in this way. Then, we applied the communicability breakdown tags of the Semiotic Engineering's Communicability Evaluation Method (CEM) [47] to interpret and organize the results. The use of CEM tags (and the breakdown categories that they correspond to) to characterize breakdowns in different contexts than originally proposed has already been done by Afonso [50] in studies of APIs communicability. Their evocative phrasing, representing deeper analytic categories, is helpful to reveal an immediate sense of recurring situations users face when interacting with the technology.

Very briefly, Communicability Evaluation tags are informal expressions used to represent the presence of communicative breakdowns divided into three major categories: Complete Communicative Failures; Partial Communicative Failures; and Temporary Communicative Failures. Failures are analyzed not only in terms of their form and content, but more importantly in terms of presumed intent (or intentions) of communicating parties. Therefore, Complete Failures are those where all parties fail to achieve their (mutual) intent. In $\mathrm{HCI}$, this accounts for designers failing to communicate their design intent and rationale to users via the system's interface and, mostly as a consequence, the users failing to communicate their intent to the system, via the interface. Partial Failures are those where one party's intent (the user's or the designers') is achieved, although at the expense of complete satisfaction of the other's expectations or preferences. Finally, Temporary Failures are those from which communicating parties (typically the user) recover in the course of interaction (e.g. through error correction, workarounds, etc.) and are able to restore the communication flow.

\section{RESUlTS}

\section{A. "What's this?" and "Oops!": Temporary failures as signs of explorations in practice and evolutionary interpretation}

As their name suggests, temporary failures happen when the communication breaks but users are able to recover and restore the flow of the interactive conversation with the system, the designer's deputy. This usually happens in three different types of situations; when user's ongoing interpretation of the system halts momentarily, when users cannot communicate back their intentions to the system, or when users simply become unable to understand the designer's deputy's signification choices [46, pp. 42-47]. For each type, there are different tags corresponding to particular different situations. We observed practically all temporary failures in our study but for the sake of space and time we will only discuss the most frequent ones and most relevant to the 
appropriation theme. In addition, we adapted the meaning of the tags according to the observed conditions.

The "What's this?" tag indicates when users look for clarification about the system. In a graphical user interface (GUI) system, this tag is typically associated with users examining the interface signs, looking for tooltips, and examining the behavior of a sign. However, in IoT cyberphysical ecosystems these options are not always available or sufficient and users tend to explore the system in practice. In this sense, the occurrence of the "What's this?" tag was often associated with the occurrence of an "Oops!" tag right after it. The "Oops!" tag indicates situations when users make a mistake but immediately fix it, usually performing an "undo" operation when available. With IoT technology, when exploring technology in practice, the result often cannot be assessed immediately after a certain operation or configuration. Often times, users had to wait for external conditions until the result could be assessed, e.g. turning the lights on or off at a certain time of the day can only be checked at that time of the day. Only then, the configuration is observed to be "wrong" or unsuitable and is undone. Therefore, the way we used the "Oops!" tag is not necessarily associated with mistakes made by users. Usually, they were associated with the evolutionary nature of users' interpretation of the technology. It will become clearer with the following cases we observed.

In a diary submission, $\mathrm{P} 3$ reported: "The smart button is used in roughly the same regard in that I use it in order to open up another application. This time it was Snapchat. I honestly cannot see myself doing this in the long run, but pressing the button and opening the application was quite fun. " Clearly, P3 did not make any firm commitment with this setting, neither was it a big necessity for him. Nevertheless, he said that it was "quite fun" to press the Flic button and open an application in his phone. His attitude was interpreted by us as an exploratory behavior, when users do not have a clear or pre-defined goal other than just to experiment the technology in action and see "what's this?" In other words, users are trying to discover what the technology means. From an appropriation perspective, users are not just trying to discover what the designer's deputy means through the message sent in the technology interface signs; they are also, and perhaps more importantly, trying to discover what the technology means for them. What the technology means for them is related not only to what the designers say through their deputy's interface, but also to what practical uses users can make of it.

After experimenting with a certain feature or setting, there were cases where users did not like the result or did not find a practical or appealing enough use case for it. In such cases, they simply reverted it and said "Oops!" sometimes replacing it by a different option. Indeed, we inquired P3 about how he was using his smart button in his final interview and he said:

[Researcher] And did you substitute the Snapchat feature or are you using both?

[P3] Yeah, yeah, no, I took out the Snapchat feature.

[Researcher] Ok. So, it was just a matter that you were looking for stuff, playing, and then you just give it a try?

[P3] Yeah, I gave it a try, something like that.

This kind of situation was frequently observed in our study. It comprises a strategy undertaken by participants to explore technology in practice. $\mathrm{P} 8$ reported another interesting situation:

[P8] In my last report I said that I set up the motion sensor to turn on the WeMo (which turns on a light) however I have turned that functionality off. When I set that up, I forgot to take into account that even in the mornings and afternoons, when it's already bright, it'll turn on the light. That is a bit inconvenient. I was trying to looking for a way to turn it on if there is motion after a certain time of day, say $6 \mathrm{pm}$ because that is when it's already dark and when I would actually want it to turn on. I'm not sure if there is a way to set up a time though.

We cannot say that P8 had made a mistake in this case. She performed an "undo" (that is, she turned off an IFTTT applet in the case above) because the result, although "correct" in a strict sense (the technology did what it was supposed to do), did not match her expectations. Actually, she refined her expectations to take into account other details and conditions she had not envisioned before. However, she could not set the system in the new way she realized would be better at the time. Then, she turned the feature off ("Oops!") and decided to keep looking for a more complete solution later. The previous configuration she had made to make the motion sensor turn on the WeMo became then a case of "What's this?" because she was actually exploring the technology in practice. Often times, it was only when the "Oops!" operation was reported that we were able to identify the exploratory nature of the previous operation as "What's this?" Sometimes, the original operation was even omitted and only revealed upon a new breakdown such as in "sorry I'm not sure whether I reported that before..." [P5].

P8 kept seeking for ways to accomplish what she had envisioned and reported trying other things. Even though she had not accomplished exactly what she said that she would like in the passage above, she incrementally learned more to the degree that she found a stable, useful, and practical use case for her. Actually, she abandoned the motion sensor strategy (more on this later) and automated her lights by renaming devices and based on the time of the day. That was the way that she found that the technology worked for her. This signals a point of appropriation, achieved after a sequence of exploratory attempts, which led to a stabilized interpretation.

We highlight two aspects regarding this kind of situations. Firstly, it indicates an exploratory attitude towards the technology that is positive from an appropriation point of view. Users actively tinker and experiment with the technology, incrementally building their interpretation of the system, what the system actually means for them. Secondly, this exploration can only be done in practice because technology is embedded into the "real world." The "real meaning" is "in the mind of the beholder" and depends on the beholder's context, previous knowledge, expectations, references, values, etc. When interpreting signs, we know from semiotic theory that this is always the case. With IoT technology this aspect becomes even more pressing due to technology being more embedded into everyday practices and thus more subject to the influence of real-world dynamics and contingencies of everyday life. Interpretation and appropriation are tightly coupled and are both evolutionary processes. We will discuss this coupling further in the discussion section. 
B. "What happened?" and "Why doesn't it?": Temporary failures as signs of communicability problems

A different kind of temporary failure is represented by the "What happened?" tag. This tag indicates that the user was not able to interpret the system's output because she or he could not see or understand the designer's deputy's communication, usually in response to her or his own actions. During some time, P1 was puzzled by his lights turning on without him understanding why. On a diary submission, he wrote: "I remember turning the light off and leaving the room. When passing by the room a little while later, the light was on in the colored mode. I'm pretty sure I didn't accidentally turn on the light through the app or anything after I left the room." Over the course of approximately three weeks, P1 faced other similar situations with his lights turning on unexpectedly:

[P1] Today, I woke up to the light bulb on. I didn't set an alarm with the Dot [...]. I called out to the Dot to turn the light bulb off. I snoozed for a bit. I woke up and saw the light bulb on again. Not sure what the issue is, Idon't sleep talk or anything, but this is a bit annoying.

In such cases, P1 is literally asking "What happened?" because he could not make sense of what was actually going on. Eventually, he figured out that the he had accidentally turned on a feature called "Day \& Dusk" in the LIFX app, which produces an automatic daily light cycle that mimics the natural light outside according to the time of the day. In this situation the user was not trying to interpret a feedback or response from the system to an operation he has just performed before, as is usually the case with more traditional interactive systems. The user was puzzled by the system (unexpected) autonomous behavior, in this case caused by his own accidental (mis)configuration. Interestingly, an expected feature of IoT-based systems is their capacity to behave autonomously (intelligently, context-aware, responsively) in different degrees. However, P1's reactions suggest that even autonomous behaviors should be intelligible by the end users in order to be acceptable. Autonomous behaviors should either follow some "expectancy rules" in order not to disrupt expectations or, perhaps more feasible, they should provide easy ways for users to make sense of what is going on and interfere, if they see necessary.

There were other similar cases where autonomous behaviors by devices puzzled the participants, who could not make sense of what happened. P8 and P11 also faced situations where their lights turned on unexpectedly. In the case of P1, we understood that he has lost sight of the fact the device was merely responding to a configuration he had done before by accident. During his interview, P1 reported that it was also by accident that he discovered what was causing the issue, when playing around with the LIFX app at another occasion and spotted that the "Day \& Dusk" feature was on. We can think about this as a communicability problem, where the system's current working configurations were not clearly communicated to the users. How to communicate effectively the current working configuration of an IoT ecosystem sounds as critical challenge to IoT technology researchers and designers, one that could help users depend not only on luck in order to recover from such breakdowns.

Slightly different situations were observed with the "Why doesn't it?" tag. This tag indicates a kind of temporary failure that happens whenever technology does not produce the expected effects in response to operations that the users strongly believe are appropriate and correct. In typical cases, users would insist in repeating an operation that would never lead to the expected result and not figure out why it did not work. In our study, we observed situations when technology failed to meet users' expectations without users understanding why. However, most cases were not the "users' fault." On the contrary, users' operations and expectations were correct, but the technology was not actually working as expected. Most frequent examples were some devices (the LIFX, the WeMo smart plug and the Echo Dot) loosing connection to the network and forcing users to perform a reset in the network router or in the device after a couple of unsuccessful interaction tries. Other examples were the Flic button failing to respond or presenting a too long delay and users repeatedly pressing it.

In such cases, users had not performed a wrong operation or had wrong expectations. Rather, there were actual technical glitches, in which case the fault falls on the designer. Technical glitches should be fixed but the heterogeneous nature of IoT ecosystems makes them more likely to happen and can aggravate them because problems may arise in very particular situations (e.g. a particular combination of network settings and devices). As a practical recommendation, we suggest that designers should invest their best efforts not only in testing and fixing these kinds of issues, but also build ways to detect and communicate the system's state to the users during use time. In other words, designers should invest in improving the communicability of the system's state. No one will ever be able to assure that a technical problem will never happen. However, designers can aid users' ability to cope with technical problems more knowledgeably by helping users answer "What happened?" before users ask "Why doesn't it?"

Both "What happened?" and "Why doesn't?" breakdowns indicate communicability problems that negatively affect appropriation by hindering users' interpretation of the technology. We will discuss them further in the discussion section.

\section{C. "I give up:" A straightforward complete failure}

The "I give up" tag represents complete failure situations when "the user is unable to understand the designer's message conveyed by the designer's deputy's interactive discourse" [46]. In these cases, users do not achieve their intended goals, either because they did not know how to or because they did not have the time, will, or patience to do so. Neither did the designers achieve their goal, since their intended message was missed. In terms of appropriation, users do not appropriate the technology.

Participant P6 reported a problem that she had had while trying to use the smart motion sensor: "One of the applets, which had the LIFX light turn on whenever the motion sensor detected movement, I had to turn off because it was firing off too frequently and changing the sensor's sensitivity made it not trigger at all! Hopefully soon I'll find a medium." At the end of the study, we asked her about this issue in the final interview and she answered:

[P6] That was funny. So, I was sitting and we had just set a bunch of commands for the light, cause pretty much the only thing I could see that had a noticeable reaction is the light so we connected a lot of things to react to the light like Alexa, [...] the Flic button for a while [...], and then the motion sensor too. So, we were saying things like 
"trigger lights off" and then it turned off in the room and we were watching a movie and I think someone's flicked, moved or the couch, like, wrinkled or something very, very subtle in front of the motion sensor and the light came on. And we were like "no, no, turn it off" and it so turned back off. And then someone left the room through a backdoor (like, there's a door in the back) and it turned on again and we were like "no!" So, they do have a sensitivity toggle, right, in the motion sensor app, like, you can slide it or something. So, I tried to lower it and when I lowered it someone walked by and nothing happened at all and I was like "ok" [laughs, frustrated face].

In this situation, we notice that the participant could not find a stable understanding of how the smart motion sensor works. She had an initial idea of the kind of movements that should be sensed by the device but soon she was faced with concrete evidence that "subtle" and even relatively distant moves (coming from the backdoor) were able to trigger the sensor. She then tried to regulate its sensitivity in the device's mobile app but was not able to make it work as intended. Eventually, she moved the device to a place where it would not bother her, a sort of abandonment. P6 "gave up" and did not appropriate the technology. Her hypotheses did not remain true long enough for her to build her own ways of interacting with the device because she never figured out what to expect from it. Therefore, she could not find a practical use case for the device.

All participants had trouble trying to use the smart motion sensor, when it came to figuring out how the device actually works and making proper use of it. P10 reported that he "panicked" the first time he left his home for a holiday and started receiving a flood of notifications from the motion sensor. He still did not know why it had triggered in that situation at the time of the interview. P5 reported a similar situation where he was not sure if motion detected was due to a maintenance visit in his room or the wind moving his blinds. P11 pointed that he expected that his two "under 15 pounds" dogs would not trigger the motion sensor. The common approach was participants eventually turning the motion sensor off after a couple of trials, either unplugging it from the power outlet, moving it to a place where it would not bother them, or turning off any automatic behavior attached to it.

Some participants (P7, P8, P11) kept the motion sensor on as an informative device that they could manually check once in a while, or still hoping that they will learn how to make a good use of it in the future. As P11 has put it:

[Researcher] But it looks like you would like to still try it someplace else?

[P11] Yeah, exactly. I can see it's useful but it's just I have too many variables going in and out of my room, with my dogs and it might interrupt some things. Like, if I have it turned on for light while I'm doing homework and then one dog comes by and turn it off, ehmmm... I think I have to mess around with it more to find a proper use.

Therefore, "giving up" is not a definitive state and is subject to users' reassessment in the future when their availability of time, will, or patience changes, or even when their understanding of how the motion sensor works improves due to external factors (e.g. talking to a friend who is knowledgeable about motion sensors).

\section{D. “Looks fine to me...": Subtler (and more dangerous) complete failures}

The "Looks fine to me" tag also refers to complete failures where both users and designers did not accomplish their goals. However, this is a trickier, even dangerous situation, because users mistakenly think they have succeeded when they actually have not, leading to misinterpretations and 'silent' failures. These can take a long time to be perceived by users, if ever, possibly causing undesired problems of varying severity. The following examples point to this kind of situations.

Participant P5 was using two location-based IFTTT applets to turn his smart light off and on when he left and arrived home, respectively. At some point during the study, he reported in a diary submission that the applets were not working as expected: "I noticed that I had misconfigured the IFTTT applets that controlled the LIFX bulb's state when I left and returned home. They [were] set to the wrong location so the light had been on the entire time I was away while I thought it was off and I was mistaking the notifications for the correctly configured WeMo applet notifying me it was turning on/off on my departures/arrivals home. So, I changed it." In the final interview, he elaborated more on this situation:

[P5] [...] Actually, in the beginning, I messed up, I think I mentioned recently, I messed up the location setting for the automatic turn on and off when I exit...

[Researcher] Oh yeah, you told that. So, you just realized at the end that it was broken?

[P5] [laughs] So, I was leaving, coming back, thinking that it was automatically turning off but it was on all day [laughs]. I thought it was so cool, I was like "oh, look it, it turns on when I get here."

[Researcher] So, how did you realize that?

[P5] I was looking through the IFTTT applets and I entered the configuration and I saw the..., because you know it tells you, the IFTTT applets say last time they run?

[Researcher] Oh, you have like a log of executions, something like that? Oh, no, no, no, you are just checking on the applet cover, I see.

[P5] On the cover, yeah, and that's where I saw for the exit one it said "never run" and I was like "what?" So, I remember I left my house and then my phone just got a notification, and I was like "oh, it's the light" but it wasn't.

From the time P5 reported he was using a location-based applet to control his light to the time he realized it was not working properly it took approximately 10 days. Other participant P10 faced a similar situation: "My location applet for my LIFX bulb has stopped working. It doesn't turn the light on when I get home and it doesn't turn off when I leave." In the final interview, P10 suggested that the root cause, in his case, was that he had used the action "toggle lights" in his IFTTT applet. Thus, the applet would fail whenever the light was off and he left home because it would toggle the light on, and then toggle the light off when he came back, working in the opposite way than expected.

These are typical situations where the user achieves some results that he believes are correct but has misinterpreted the system, not realizing the result is not the expected one. To 
some extent, appropriation corresponds to the ability that users have to rely on and resort to the technology at hand to accomplish their goals. Using abductive reasoning, users come up with their own explanations about how the technology works and about the situations they face, which will remain true until evidence of the contrary is found. Originally, the "Looks fine to me" tag points to situations of complete and definitive failures, which may, nevertheless, make local sense in short term observations or task-oriented systems. However, we observed that these situations can be temporary. According to the abductive reasoning process, interpretations are always subject to revision and can be fixed when wrong, even if after a long time. A necessary condition to make users revise a certain interpretation is a surprising fact, something that challenges their current understanding of the technology. Actually, the situations faced by P5 and P10 correspond to this moment of facing a surprising fact. We can name this situation and propose the "Uh-oh!" tag to refer to the precise moment of users realizing that they are wrong, whilst "Looks fine to me..." refers to the previous moment when they have made a mistake. These precise moments of users realizing that they are wrong are actually the repairs of the previous "Looks fine to me..." breakdowns, the repairs that made the breakdowns visible to us.

If a "Looks fine to me..." breakdown takes place, we would like them to be followed by an "Uh-oh!" repair as fast as possible. In best cases, users will be able to diagnose and fix the problem, such as with P5. In worst cases, a "Looks fine to me..." episode will not lead to an associated "Uh-oh!" and will have negative consequences, such as wasting energy in our cases. Situations like these should be addressed by good HCI designs, which is more difficult in IoT contexts due to the distributed and cyber-physical nature of the IoT devices and the derived composite systems. We will discuss more about some implications of this kind of issues in the discussion section.

\section{E. “Thanks, but no, thanks:” Partial designers' failures of users declining designed affordances}

The "Thanks, but no, thanks" tag points to situations in which users have declined designed affordances present in the system [51]. An important characteristic of this kind of breakdown is that they are partial failures because although users are usually able to see the design alternative and succeed with their goals using a secondary alternative or workaround, from the designer's perspective it represents a considerable amount of effort that has been wasted in designing features that turn out not being used.

Participant P7 has tried to use automatic routines and schedules for her smart plug and for the smart light in her room. However, she turned off these automatic behaviors soon, preferring to control the devices manually. When asked about these situations during her final interview, this is what she said:

[Researcher] You said you don't like the schedule thing very much, you don't like the location very much. So, looks like you don't like automatic stuff very much, is that correct?

[P7] I like the idea a lot but then just actually doing it, I just get annoyed at the same time. Like, the thing automatically going on. I think I like control, like, I'm controlling everything myself. Yeah.

\section{[Researcher] Ok, but still you like the idea?}

[P7] I love the idea of automatic. It's just actually in real life, I guess, like, in my head I like it but then "next, not right now" [laughs].

One of the most immediate use cases for smart IoT technologies is automating behavior, in which things react to schedules, sensors or events without human direct intervention, so to speak. Indeed, many features in the devices themselves (e.g. schedules and routines) and in auxiliary apps (e.g. IFTTT applets) are made available precisely for this purpose. Nevertheless, the user had deliberately rejected the automatic capabilities provided by the technology designers. From an appropriation point of view, P7 has successfully appropriated the technology. She perceived, understood, tried, and finally consciously rejected a designed affordance. However, from the designers' perspective, these situations challenge the very assumptions designers have made when designing the technology (e.g. "users like and want automatic behaviors").

Other participants reported situations where features available for a particular purpose were not adopted. For example, P3, P4, and P11 did not use the recurring clock alarms available in the Echo Dot and preferred to repeatedly and "manually" set the alarm for the next morning every night. Unlike with P7, with P3, P4 and P11 we were not sure that participants were aware of the provided functionality of "recurring alarms" in Alexa. During the interviews, some participants told us that they just preferred to set the alarm every night because their schedules varied too much. However, we did not ask them explicitly if they were aware of Alexa's recurring alarm feature, which is something not very straightforward to find in the Alexa's mobile app or to perform by voice. In these cases, the breakdowns got blurred with the "I can do otherwise" tag (next sub-section) in our analysis. Originally, "whereas 'Thanks, but no, thanks' is an explicit declination of some affordance, 'I can do otherwise' is a case of missing some intended affordance" [45], but our data have not captured this separation clearly in every case.

\section{F. "I can do otherwise:" Partial designers' failures; Interesting appropriations by users}

The "I can do otherwise" tag is similar to the previous one in the sense that, in both situations, designers failed to communicate (the value of) their design intent and rationale to users, but users could still accomplish their goals. Originally, the "I can do otherwise" tag indicates users missing an affordance and achieving their goals in sub-optimal ways because of that. However, we are using the "I can do otherwise" tag to indicate all the situations where users explicitly created their own ways of performing a certain task. Sometimes, this happened because of a missing affordance, that is, the user was not aware of a feature provided by the designer. Other times however, we observed situations where there were no originally provided alternatives to fulfill what the user wanted, leading them to customize the system in very personal ways. These situations represented the situated adaptations that constitute the hallmark of appropriation. Both cases will be further discussed separately.

As an example of users missing an affordance, the D-Link smart motion sensor companion app presents features for manually enabling/disabling the motion detection and even defining an automatic schedule for that. However, all except one participant seemed to have missed these features (only P8 
reportedly tried to use the motion sensor schedule). Participants P3, P5, and P10 did not use the motion sensor app for enabling and disabling the motion detection, preferring to plug the motion sensor in the smart plug in order to turn it on and off and achieve the same goal. These are typical examples of the "I can do otherwise" tag because users seemed to have missed the available feature. Usually, this points to communicability problems in the systems' interfaces that caused users to miss some affordance and overcome it with some other sub-optimal way of achieving the same goal (in this case, using another device to accomplish what could be done with the smart sensor alone). From an appropriation point of view, users are appropriating the technology in the sense that they are accomplishing practical things as they can, which is positive in itself. However, the appropriation could be improved by the design of an improved communicability of the interfaces.

The case of end users building functionalities not originally provided by the designers is more interesting. Participant P6 referred to herself as being obsessed about the weather and thought about using the Flic button with this purpose since the beginning of the study: "I'm really obsessed for the weather especially here to have, like, a little weather summary or something. "She has struggled to make the Flic produce this outcome and eventually she was able to accomplish it "in a bit of a roundabout way," as she described it in the final interview:

[P6] And I actually did get it to pull up the weather but it was in a bit of a roundabout way because it doesn't actually have a command for it. So, instead you can, ehmm, when you push, one of the functions of it is that you can call up a web page. So, I just copied the URL for the weather in Irvine. So, I hold down the button it would open up the weather for Irvine right now and I was like "Oh!" [impressed and happy]. I mean, that's kind of what I wanted so it ends up working anyway. So, it's really good. I used that, once it actually started working, I was using that all the time.

Another participant P5 described a similar situation where he wanted to use a daily schedule for his smart light based on a native feature available in the LIFX mobile app called "Day \& Dusk." As mentioned before, this feature produces a daily light cycle according to the time of the day, smoothly transitioning from completely off, then a bluish white light in the morning to a warmer, yellowish light at night and then turning off again. However, in his personal view, he wanted to use different colors in his daily cycle and the native feature only allowed him to choose between shades of cold and warm whites. His solution was another "roundabout" where he combined the "Day \& Dusk" native feature with some IFTTT applets to slowly change the light color in order to produce the desired outcome.

What is interesting in these situations is that users have created their own "roundabout ways" to achieve their desired goals, overcoming perceived or actual limitations of the technology. By means of their adaptations, often "roundabout ways" or workarounds, users say they can do otherwise. Perceived limitations (e.g. P3, P5, and P10 plugging the motion sensor into the smart plug in order to be able to turn it on and off in spite of the motion sensor providing features that allowed this configuration) were a consequence of user not being aware of a feature or affordance originally provided by the designer. They correspond more precisely to the original meaning of the "I can do otherwise" tag and usually point to communicability problems regarding the missed affordance. However, sometimes, the desired functionality was actually not available, to the best of our (and users') knowledge and users dealt with actual limitations of the technology (e.g. P6's Flic weather report "in a bit of a roundabout way" and P5's "Day \& Dusk with colors" feature). Whenever users develop their own workarounds to overcome actual limitations of the technology users are not exactly saying that they can do otherwise (there is no designed way to contrast with the "otherwise"); they are saying more, that they can work around some actual limitation of the technology. Therefore, we propose the new tag "I can work around it" to designate these situations. This new tag does not point to communicability problems but to situations of use that were not anticipated by the designers.

In terms of appropriation, these situations represent the most interesting cases, where users have invested a great amount of their time and effort building their own customizations to accomplish practical goals. Therefore, we can suppose that these practical goals were considered valuable by the users. The "I can do otherwise" tag reveals potential communicability problems which can be solved by designers with better interface design (e.g. exposing or emphasizing a hidden affordance). The "I can work around it" tag means more than that. The fact that users have appropriated the technology to the point of accomplishing goals not originally anticipated by designers shows a remarkably high degree of appropriation. This situation will be further discussed in the next section.

\section{DISCUSSION}

The breakdowns described in the previous section point to some important aspects about how the appropriation process unfolds. These aspects can contribute to our fundamental understanding of appropriation of IoT technology and the consequences to the design thereof.

\section{A. Abductive Appropriation}

Appropriation unfolds over time, as users explore, learn and get familiar with the technology, to the point when they start to incorporate it into their practices, by making more consistent use of it, often adapting themselves and their practices to the technology [35]. This process includes learning the technology, envisioning possible usages for it, and developing an overall attitude towards it [52]. There is an intricate relationship between using/learning the technology and practicing/reflecting about what the technology is intended to support. The interplay of these underpinnings becomes clearer when users adapt the technology to their practices but can never be really separated. One way to untangle this intricate network of relationships and activities users perform is to look at appropriation as a state rather than a structured process. In a previous version of this work, we proposed that "appropriation corresponds to the stable state which allows users to organize a rich and productive set of interactive discourses with the technology at hand" [1]. We now extend this definition to the following: appropriation corresponds to the stabilized state of interpretation which allows users to organize a rich and productive set of interactive discourses with the technology at hand. This definition is more precise and potentially more useful because it splits the problem into two; the problem of interpreting 
technology and the problem of supporting the design of rich and productive interactive discourses with it.

Note that a rich and productive set of interactions is not the equivalent of correct interactions, expected/predicted interactions, proposed interactions, etc. All we can say is that rich and productive interactions are a subset of possible interactions (whether the designers of the technology were aware of such possibilities or not). Rich and productive interactions necessarily make sense (and therefore have meaning) to the users, regardless of whether they make sense for anybody else, including the designers of the technology. Therefore, rich and productive interactions are the result of satisfactory interpretations of the technology in the sense of users finding "the real meaning" of the technology for them. For successful appropriation to happen, it is not only necessary that users understand the designer's message embedded into the system. Moreover, users also need to understand how to make practical use of this message in their own contexts and to accomplish their own goals.

Satisfactory interpretations are not the equivalent of definitive interpretations. Following Peirce's theory of abduction and its role in semiotics, satisfactory interpretations are those that resist the test of abductive reasoning. In abductive reasoning, the "reasoner" (in our case, the user) generates hypothetical general principles that explain some observed phenomenon and resist the reasoner's limited testing of the principle, in contingently available cases. Because such testing does not cover all possible cases to which the hypothesized general principle actually applies, the reasoning may prove to be incorrect in the presence of 'future events' (which leads to a revision of the principle, more testing, and new provisional conclusions - or interpretations - that can be revised because of counter-factual future evidence). Therefore, users can build a sense of "appropriation" that can resist for longer or shorter time, but eventually prove to be incorrect or insufficient. No appropriation is definitive, but lasts until evidence is found that falsifies or enhances the previous understanding users have built in their minds. A current state of appropriation is thus linked to a current a state of interpretation of the technology.

However, this state should be minimally stable in order to allow users to build interactive discourses upon it consistently, which is not possible over an ever-changing ground. If users' assumptions are not correct, attempts to use technology in practice will fail quickly, forcing users to review their hypotheses. A breakdown is the moment when the user realizes his or her current interpretation state is wrong or insufficient, triggering the pursuit for a new one. The "stabilizing process" is a series of mental (cognitive), physical (interaction) and social (e.g. collaboration with other users) activities that will follow a breakdown. If successful, users will evolve from a prior state to a richer one, in the sense of enabling other richer interactive discourses. If users are not able to sediment a new understanding, they may either remain stagnated in the same appropriation state (e.g. doing what they already knew in the way they knew), if some practical use of the technology is possible, or abandon the technology, otherwise. Both situations can be temporary, since users can never be stopped from revisiting the technology and their thoughts about it. We call this characterization "abductive appropriation" because this description resembles the abductive reasoning process, which is deeply related to Peircean semiosis. This description is depicted in Fig. 1 showing a sequence of appropriation states triggered by a breakdown and the following stabilizing process in-between two states.

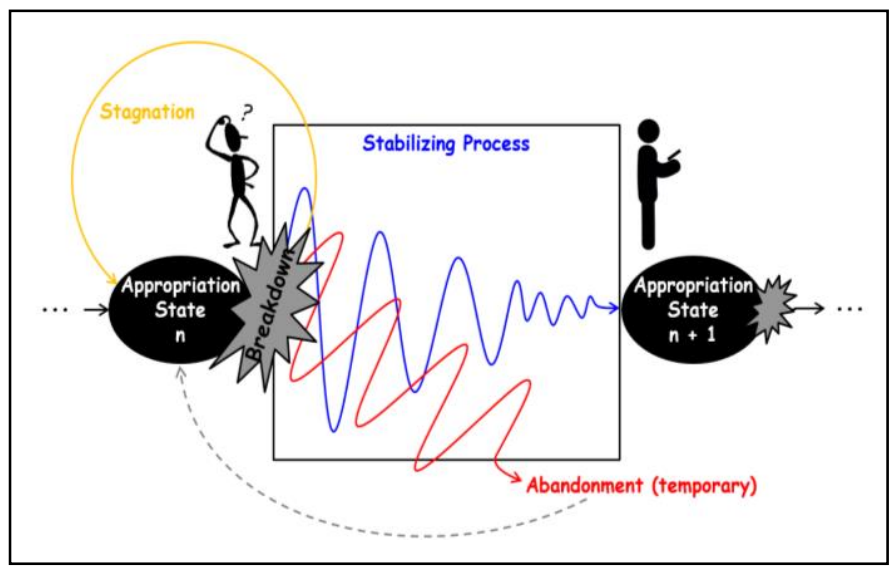

Fig. 1. Appropriation as states and possible events or processes in-between them.

Notice that this framework can describe different appropriation states in a generalized way. For instance, the initial contact of a user with the technology is an initial appropriation state grounded in the interpretation she or he has about it beforehand. This interpretation includes any previous knowledge or belief about the technology, including those they have before having any actual contact with it, what Karapanos et al. called anticipation in their framework [38]. Any current interpretation generates certain expectations. Breakdowns correspond, precisely, to mismatches between users' expectations and what they observe in practice. In the abductive process outlined above, a breakdown triggers a revision in users' interpretations, just like the situations we observed in our empirical study. In that sense, breakdowns are very important to appropriation and will discuss them further ahead.

\section{B. Breakdowns as Signs of Appropriation}

In our framework, breakdowns are a natural and unavoidable part of the appropriation process and mean two things. In terms of communication, breakdowns are actually users talking to themselves, so to speak, in an unarticulated way, about what they cannot tell the system explicitly, as an instinctual attempt to repair the communication flow. In that sense, the CEM tags are valuable because they "put words in the user's mouth" [46, p. 37] and by doing so they help us understand what users are trying to say. In terms of appropriation, the breakdowns we observed reveal certain appropriation states. In other words, breakdowns stand for particular states of appropriation in the abductive framework proposed above. According to Peirce, "a sign is something that stands for something else to someone" and breakdowns are thus signs of appropriation (states) to us. Table I summarizes and groups the observed breakdowns and tags described in the previous section. The fourth column describes how each tag can be understood as signs of particular states of appropriation where the users are in. This relationship will be further discussed in the following paragraphs. 
TABLE I. SUMMARY OF OBSERVED BREAKDOWNS, TAGS, AND THEIR RELATION TO APPROPRIATION STATES:

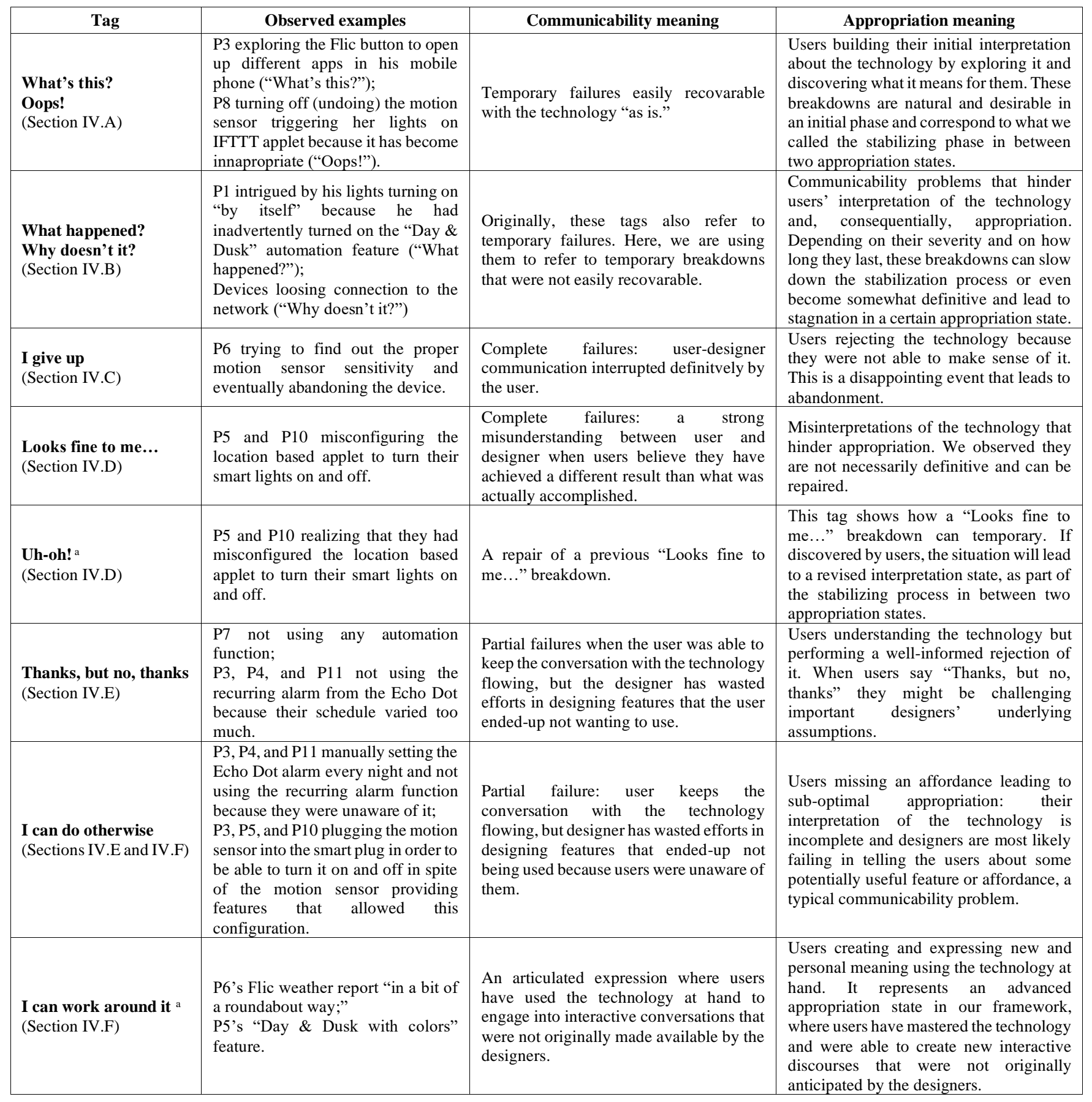

a. Tags that are being proposed by us in this paper and are not original CEM tags. As can be noticed, they are not necessarily communicability breakdowns, but should be understood here solely as an evocative phrasing that represents recurring situations users face when interacting with IoT technologies that are relevant to our appropriation framework.

“Oops!" and "What's this?" situations (Section IV.A) correspond to the stabilizing process of users interpreting the technology by exploring it in practice. The "real meaning" of technology will unfold over time and will be continually (re)assessed in situated practices. Indeed, it cannot be otherwise because according to semiotic theory, human semiosis is unlimited and cannot be prevented or limited. Every new encounter with a sign will trigger new interpretations of it. For example, everybody knows what turning on a light means. However, turning a light on when it is bright (and a waste of electricity) is not the same as turning it on say, after $6 \mathrm{pm}$. Users make sense of the "real meaning" of a sign in the "real world" because the "real meaning" is influenced by the context and circumstances in which the sign is interpreted. Moreover, meaning evolves, such as the automation rule quickly becoming inappropriate for P8 just after the first try. In that sense, users' interpretation of technology is evolutionary and evolves not only because they learn about the technology but also because they learn about how the technology fits into their contexts and practices. "What's this?" and "Oops!" breakdowns indicate intermediary steps of this interpretive process. They tend to diminish when users find a stabilized interpretation that is useful for them. Only then appropriation actually happens, when this learning stabilizes and materializes in the preferred 
use cases and corresponding settings and configurations of the system. At this point, users reach a new state of appropriation.

Sometimes, users' interpretations do not evolve and halt in a certain state for a very long while. We observed situations like these with the tags "What happened?" and "Why doesn't it?" (Section IV.B). Users getting stagnated in the same appropriation state is not necessarily negative if the current state is satisfactory and fulfilling for them. However, the situations described in section IV.B represent negative experiences where users either took too long to make sense of technology or were literally kept doing what they could to cope with some technical glitches they faced. We associated both problems with communicability problems and we will discuss some design implications related to them in the next sub-section. Depending on the severity of the breakdown and the time users take to recover from them, they can lead to the next case.

"I give up" situations (Section IV.C) correspond to those episodes where users were unable to stabilize their understanding about the technology. Sometimes they left the motion sensor underused (stagnation), other times they just left it behind (abandonment). As participant P6 reported, she tried different settings in order to make the motion sensor work as she intended. These attempts were triggered by the breakdown of the motion sensor starting to behave differently from her expectations (that is, reacting to subtle and distant moves), a kind of "What happened?" breakdown. She explored the device and took a "trial and error" approach by selecting different sensitivities. Her primary goal was to adjust the sensor's behavior at the same time that she was making sense of how the sensor worked, making and testing hypotheses. Nevertheless, she could not find a minimally stable interpretation of the system and eventually gave up. The time and effort invested in this endeavor were proportional to her available existing knowledge, will, and patience she had at the time.

"Looks fine to me..." situations (Section IV.D) were recoverable, at least to $\mathrm{P} 5$. The breakdown, in his case, was caused by his own misconfiguration of the IFTTT applets he was using to automatically turn his light off and on when he left and arrived home, respectively. However, he was misinterpreting his light's state and the notifications from another applet as evidence that his light-automation applets were working correctly. The breakdown was fixed when he spotted a sign in IFTTT that the applet had never run. He was then able to investigate deeper, make sense of what was wrong and correct the applets, leading to a new stabilized state where he could rely on this automatic behavior and also on the tools he used to achieve it, such as IFTTT, the location trigger, and the light responding to it. Although recoverable, "Looks fine to me..." breakdowns indicate difficulties participants had when assessing their smart technology behavior. In terms of our appropriation framework, this means users found themselves inside a wrong interpretation state which can last indefinitely, until they realize it or a new breakdown happens that forces them to revisit their understandings. In case users realize their mistake, they will try to repair it. We propose to name such events with a new tag called "Uh-oh!", which is actually a repair that reveals the occurrence of a prior "Looks fine to me..." failure. Repairs are typical events associated with breakdowns and misunderstandings in communication [5], [6]. How to study breakdown repairs within the more abstract classification and categorization system of communicability breakdowns, of which the tags are just the most superficial sign, should be object of further research.

In "Thanks but no, thanks" breakdowns (Section IV.E) users deliberately reject some designed feature or affordance of the technology. They correspond to successful appropriation by the users when they find their own way to make practical use of the technology after experimenting with alternative options (e.g. manually controlling their stuff at will instead of automating, such as P7). In our appropriation framework, these situations signal a clear case where the technology means different things to the designers (who say they think "this feature is valuable" when they invested in designing it) comparing to users (who might just not mind). However, this will only be the case if and only if users' interpretation and decision (of not using some feature) is well informed. Otherwise, it might be the different case as following.

"I can do otherwise" breakdowns (Section IV.F) are the richest ones from an appropriation perspective. We observed users do otherwise in two different lines of action. First, users may simply not be aware of an affordance or feature provided by designers, corresponding to the typical traditional situation represented by the "I can do otherwise" tag. This was possibly the case with the Echo Dot's recurring alarms (blurred with "Thanks but no, thanks" tag in Section IV.E) and with people using the WeMo smart plug to enable and disable the motion sensor (Section IV.F). Again, these breakdowns point to communicability problems in the interface to be discussed in the next sub-section.

A second and different type of situation occurred when users dealt with actual limitations of the technology, that is, when they wanted to do things that were not originally provided by designers. This was the case with the Flic weather report "in a bit of a roundabout way" by P6 and the "Day \& Dusk with colors" by P5 (Section IV.F). From an appropriation perspective, these situations are remarkable and we propose the new tag "I can work around it" to describe them. Again, we need to conduct further studies in order to accommodate this new tag into the relatively mature communicability breakdowns categories and abstractions originally proposed by the CEM. In a CEM-like breakdown analysis, who is failing in such situations? We think that such situations point to new design opportunities that were not anticipated by technology designers and represent opportunities for improving the technology. In that sense, this new tag corresponds to a feedback from the users to designers, a sort of inverse metacommunication message from users to designers about how users actually want to use the technology. Therefore, the failure falls in the designers' side, due to their inability to anticipate some user need. However, we can reasonably assume that a designer (and consequently the system they design) will never be able to anticipate every need of every user, an idea that is in the root of End-User Development (EUD) [53] techniques and approaches. As we said in the beginning of this section, every breakdown is a sort of unarticulated talk-back from users to designers. Therefore, the failure is actually the designers' inability to listen to users' talk-back, something that computing technologies are currently not designed to support. A question we may raise then is whether computing technologies can support users talking-back to designers and how? "I can work around it" is an extreme case where users actually articulate a clear talkback using the language of the technology they have mastered. 
"I can work around it" situations represent the strongest sign of appropriation where users have mastered, modified, and re-signified the technology. It is an advanced state of appropriation in which users produce new interactive possibilities not originally anticipated by designers and engage in richer and personally meaningful conversations with the technology. It is very important that these situations be detected and deeply understood from both a research and a practical design perspective. Applying our evolutionary appropriation framework, it means users have passed by several intermediary states to get there. According to Carroll's appropriation cycle [35], these states can be very helpful when designing from appropriation if they are seen by the designers. Some questions we may ask, why some users reach this point and others not? What in technology design can favor or hinder this process? How designers can be reached and effectively listen this talk-back? We do not know how to answer these questions now.

Finally, we should say that there are appropriation states that are not signaled by breakdowns. For example, if everything is working fine, it means users are able to use the technology "as is" and are probably satisfied with it. Therefore, no breakdown happens. Indeed, we observed several situations where this was the case:

[P1] One use case that I use quite often is using the Dot to turn on/off the LIFX light bulb and adjusting the light bulb's color. So, when entering/leaving my room, I would call out "Alexa, turn off [or on] the light." I had set the light bulb's name to "light" within the Smart Home tab in the Alexa app.

\section{[P5] I use Alexa almost [all] day to play music.}

In such cases, the absence of breakdowns is a sign of a certain stabilized state of appropriation, one where users are engaging in productive interactive conversations that fulfills their needs and preferences. This is certainly a sign of a successful technology design by designers, where their evaluation of users' goals, needs and preferences match users' actual ones. In Semiotic Engineering terms, users' goals, needs and preferences and the system's interactive logic are the gist of the metacommunication message. Of course, absence of breakdowns may happen when users are not even engaging in conversations with the technology and this is a completely different case (perhaps due to lack of interest, rejection, or after abandonment). However, when explicitly reported that this was not the case, absence of breakdowns indicates a good communicability design.

\section{Semiotic Qualities for IoT Technology}

As mentioned before, "What happened?", "Why doesn't it?", and "I can do otherwise" breakdowns can point to communicability problems of IoT interfaces regarding different aspects of the technology. Similarly, "Looks fine to me..." situations usually represent serious problems because users end up thinking that they have succeeded when they have not. In the cases we observed, a relative success by the user was proportional to the users' knowledge, skill, will, patience, and maybe even luck, factors that technology designers cannot (should not) rely on and resort to. The definition and characterization of appropriation we propose does not exclude misinterpretations and mistakes. Rather, it embraces them as natural parts of human semiosis and abductive reasoning. Consequently, an important part of interaction design for appropriation is to support "abductive reasoning" and "knowledge revisions" by users. That is, technology should both: (i) help users assess their current knowledge about the technology; and (ii) support the revision of their interpretations of the technology, so that "rich and productive interactions" can be resumed whenever the user him/herself realizes that he or she is wrong. Not easy goals though. In the following, we discuss some initial implications derived from our findings that can be useful to inform designers towards better interactive and appropriable IoT technology.

IoT technology currently available comprises, in general, different specialized smart devices that can be combined with each other in order to produce more complex behaviors. In that sense, the "system" or the "application" is distributed into different devices and apps that have different responsibilities, such as the smart light to produce a desired output in the environment, the mobile phone for controlling the light or sensing the location, and auxiliary tools such as IFTTT that allows the combination of the previous two. As a consequence, setup and interaction are spread in different interfaces. Everything together demands from users the ability to cope with multiple components, often having to predict the outcome of certain combinations of settings in different times and circumstances. This scenario is more complex than traditional interaction (e.g. desktop and mobile apps) and designers should seek for ways to alleviate the arising challenges.

An immediate consequence is that successful interpretation of technology will depend not only on the design of each component in isolation, but also on the quality of the system comprised of components put all together. From a system's perspective, users deal with multiple technological components. Interactively speaking, users deal with multiple interfaces exposed to them. Semiotically speaking, users face different meaning systems, each one with its own sets of signs, "linguistic" rules and metaphors. This calls for, at least, two new design qualities that we propose that should be pursued in the design of IoT technologies: cross-interface consistency and cross-device coherence, illustratively depicted in Fig. 2.

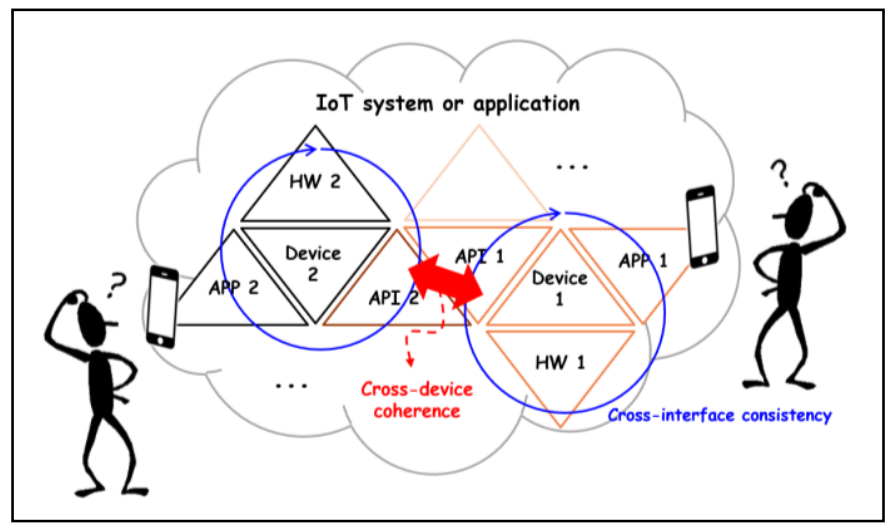

Fig. 2. The scope of cross-device coherence and cross-interface consistency.

Cross-interface consistency relates to consistency between different interfaces of a single device. A study about the Philips Hue smart device [54] suggested that IoT devices currently available follow a general pattern of architecture composed by the device hardware (HW), a mobile app (APP) for remote and advanced control of it (sometimes, also a similar web app with the same purpose) and a public API for 
integration with other devices and apps. Users interact with all these interfaces: with the mobile app through a usual mobile interface, with the hardware (e.g. placing the device somewhere, watching its color, etc.), and also with the API indirectly when they use an auxiliary tool that plugs to it, such as IFTTT. We propose that all three components should communicate a logical and consistent message in order to promote the correct understandings of the system. In "real life," inconsistencies may easily emerge because we know designers are often split into different teams depending on the component to be developed. Moreover, the "substratum" of each interface is different in nature and in the types of configurations and affordances they allow [55].

As an example of this principle, take the motion sensor and the situation described in section IV.C where users could not make sense of the smart device. Fig. 3 depicts two interfaces of this device, namely its mobile app and its hardware, displaying the sensitivity toggle in the mobile app which P6 used to try to regulate it. We cannot blame her for not making sense of how it works purely based on the information provided. Technically, the device, which plugs into any regular power outlet, is based on Passive InfraRed sensor (PIR) and can detect movements in a range of 8 meters (26 feet) from it [56]. All the feedback users have is a subtle blinking led in the hardware whenever movement is detected and the time of detection in the app screen, usually after some delay. Cross-interface consistency in this case, for example, would call for a clear visualization of the detection field of the sensor consistent with the sensitivity toggle in the app. Innovative interface technologies which are so closely related to the IoT, such as projections or holograms, could be used so that this feedback could be realistically provided by the hardware interface in the physical world and not through simulations in the app. In this way, both interfaces would contribute to communicate to users what sensitivity actually means, promoting better interpretations. That would be a concrete example of good cross-interface consistency.

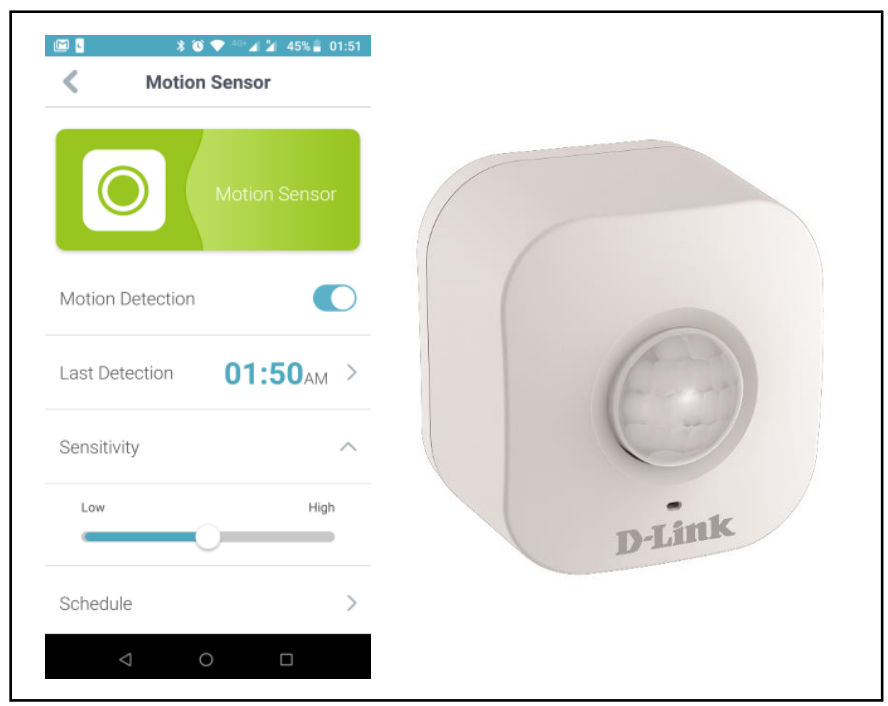

Fig. 3. The "myDLink Home" mobile app at the left (Android version) and the myDLink Wi-Fi Motion Sensor (DCH-S150) at the right.

Cross-device coherence can be even harder to achieve. It means not only that a device be logical and consistent individually but also that, when combined with others devices, they all together form a minimally unified and harmonic whole. Differently from traditional software running on a desktop or mobile, IoT devices do not have an operating system (OS) able to provide a set of functions and interaction standards that developers can use and follow in order to facilitate users' interpretations based on the OS's language, features and libraries. This means that each device, often from different manufacturers, has its own semiotic language, the set of meanings and representations with which they are designed and implemented, and that this language is much less constrained than in other settings (by the lack of a common OS standard and a much more open space for different hardware and shapes, for example). The question to answer is how is each device's semiotic language able to compose with the others in order to build a well-formed whole?

For instance, the possible cause for the occurrence of the "Looks fine to me..." breakdown with participant P10 (section IV.D) was the "toggle lights" function mentioned before. When the user is not viewing the current light state (that is, not looking at the light or at an indicator of the light's state in the mobile app, for example), it is impossible to determine the final outcome of this simple operation. Investigating this situation deeper, we can notice that despite there is an action called "toggle lights" in IFTTT (to be used with a location trigger in an applet, for example) there is no toggle function (in the sense of a "blind" toggle) in the LIFX mobile app because the user can always see the current state of the light in the app screen. Therefore, we may conjecture that this situation, though not caused by, could be prevented if IFTTT, the third-party app, followed a sort of coherence rule with the native LIFX app. Going one step further, this coherence rule could be enforced (or at least encouraged) by the LIFX component that lies in-between LIFX and IFTTT, which is the LIFX API. Indeed, there is a "blind toggle" function in the LIFX API for which the response is just an "ok" status (Fig. 4). Perhaps the designers of this API could have reconsidered the design of such a function if they had means to evaluate their decisions better. Very simply, it could provide a more complete response such as the resulting on/off state of the light that could be then communicated to the user by IFTTT (and any other third-party app communicating to it).

POST https://api.lifx.com/v1/lights/all/toggle

\{

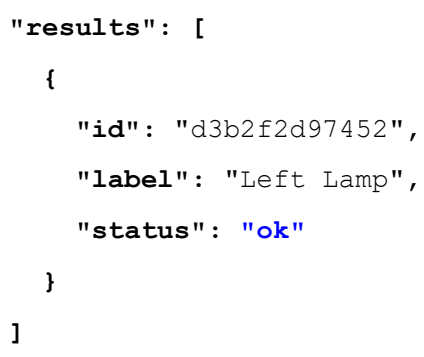

Fig. 4. The LIFX API response in JSON for the "toggle lights" function. The response does not tell API users if the light ended up on or off after the operation (Link: https://api.developer.lifx.com/docs/toggle-power, visited November $\left.4^{\text {th }} 2019\right)$.

The qualities we are proposing here call for new evaluation methods and design practices that clearly require further research. In that sense, Semiotics and Semiotic Engineering are a useful and powerful theoretical foundation because both naturally deal with the linguistic structure and "materials" (signs) of interfaces and systems, which end up shaping end users' interactive discourses and the 
(dis)appropriations that follow from them. For instance, with a different goal, Maués and Barbosa also took a semiotic approach and proposed the concept of cross-communicability, defined as "the system communicability across platforms," and an inspection method to evaluate it [57], [58]. Crosscommunicability is close to the concepts we are proposing here but differs in the sense that it was originally proposed to address challenges of users interacting with the same application in different platforms (e.g. YouTube in a web browser, a mobile phone, and a smart TV). In this context, each platform app can be used in isolation because the core application functionality is replicated and they do not depend on each other. The concepts we are proposing here are oriented to address different components not to be used in isolation because they only make sense as a system when they are used all together (in the case of cross-interface consistency, they do not even work one without the other). Similarly to Maués and Barbosa [57], [58], we find that both the communicability concept and semiotic methods can be extended to address the cross-interface consistency and crossdevice coherence of IoT technologies. Further study of these two qualities requires studies specifically designed to this end and is left for future work.

\section{LIMITATIONS \& FUTURE WORK}

Qualitative research is primarily based on interpretive analysis and therefore driven by researchers' interpretation of the data. In that sense, the meaning of each empirical evidence and the very choice of what constitutes an empirical evidence is biased by the researchers' own background and view about the phenomenon of interest. Researcher bias is unavoidable and not necessarily a threat to scientific validity if findings and conclusions are shown to be logically grounded either in evidence, in accepted theory or in both. In this paper, we strove to provide a sound account grounded in both empirical evidence and in Semiotic Engineering as our stablished theory of choice. As with any qualitative research, findings and conclusions need to be used wisely, especially in regards to the transferability of results to other contexts and purposes [59]. Nevertheless, a strong theoretical grounding can facilitate and leverage the application of our framework to other systems or even contexts, in the same way that we used the Communicability Evaluation Method tags originally proposed for GUI applications in the IoT domain.

Our method uses the same tagging principle and most of the tag classes as the Communicability Evaluation Method (to "put words in the users' mouths" [46, p. 37]), but follows a different procedure. Breakdowns were indirectly observed by means of users' self-reports. Although we believe in the ability of this approach to capture what was most significant to participants, other breakdowns may have gone unreported and, consequentially, unnoticed to us. What was reported was also used as a criterion of significance to the participants. We highlight that we focused on understanding users' overall experience and the appropriation of the technology by users as a cumulative result of breakdowns. However, this approach is probably not best suited for fine-grained evaluation of the interaction and individual interfaces of the devices and apps.

The study design had to be guided by several practical decisions. The set of IoT devices and the population of users of our study were chosen for practical reasons. Different IoT devices and apps could entail different experiences to users. Moreover, users' technology background could also have masked problems they were able to overcome by themselves.
In addition, we made use of weekly tasks proposed to participants in order to minimally expose them to some of the technology possibilities and enrich the collection of data. In this sense, tasks ended up "teaching" participants about the technology and potentially fastening appropriation, since participants were exposed to features and resources that they would have to find out by themselves otherwise. Nevertheless, tasks were not evaluated in any sense and we emphasized that participants were always free to do what they wanted with the technology. Furthermore, the strategy of compensating participants with the devices was taken as a way to increase their sense of ownership since the beginning of the study. Therefore, although possibly influencing appropriation speed, we believe that we minimized our influence on their personal judgments about what to use and how. The fact that many participants reported abandoning what they did in the tasks quickly after doing it supports this vision.

Our study was conducted in the smart home domain. There are several other IoT applications and domains which can emphasize or deemphasize certain aspects of our findings. For instance, in organizational (e.g. a company) or public settings (e.g. smart cities), users can develop a less personal attitude towards the technology and a different appropriation account may be more useful or appropriate depending on which aspects of the technology one is interested in. Nevertheless, the appropriation framework we propose here is applicable and can provide useful insight whenever people interact with IoT technology, which covers practically all domains and applications except "pure" machine-to-machine IoT (if there is any). It is left to future work the application of this framework to other IoT domains and applications.

Another interesting line of future work is to develop the understanding and application of the new tags "Uh-oh!", as breakdown repair signs, and "I can work around it," as the most advanced appropriation state that we found. At this point, our research gets closer to End-User Development (EUD), a research field focused on the study of technologies that are tailorable by the end users [53]. Our plan now is to reconstitute each users' appropriation path as a sequence of related breakdowns composing representative episodes. This episodic view over time may clarify different appropriation states that users go through when appropriating the technology. By understanding these paths of the most successful users, we could, potentially, identify relevant factors that empower users to "work around" whenever they want. On the opposite extreme, the path of the users who did not go far in terms of appropriation states can, potentially, reveal factors that hinders this process.

Finally, we are leaving to future work an investigation of the interpretation of technology in this framework. The abductive appropriation framework sets the stage and the semiotic qualities we propose here just scratch the surface of a much larger problem of understanding and supporting people's interpretation of (IoT) technology. In that sense, our research goes in the direction of Pragmatics, the component of the theory of signs specifically concerned with the relation of signs to their interpreters (that is, the people who interpret the signs, the sign users) [60]. In Linguistics, Pragmatics studies concepts such as context, coherence, and relevance [61], among others, and seem to align with the problem of technology interpretation we start to address here. 


\section{CONCLUSION}

Appropriation of IoT technology is an under-explored topic. In this work, we reported findings from a diary study with novice users of smart devices over the course of 4 weeks. Appropriation deals with the way people adopt, use and adapt technology over time. In this sense, appropriation encompasses different interactions that users perform for different purposes at different times and in different situations, configuring and adapting the technology. To a great extent, appropriation corresponds to the final result of all of the user's interactions, making it difficult to evaluate and design for it. Using Semiotic Engineering as a theoretical lens to qualitatively analyze the data, we observed patterns of communicative breakdowns derived from the Communicability Evaluation Method and related them to the appropriation of technology by users. These patterns of communicative breakdowns indicate significant stages of the appropriation process. In particular, they signal different states of interpretation users develop about the technology at different times.

Appropriation has been identified as an important component of positive user experience and is related to how technology becomes meaningful in one's life [38]. In itself, user experience is related to the way users perceive and recall their actual experiences with technology, which invariably pass through the filters of users' personal interpretations [62]. Our findings suggest a clearer relation between these interpretations and appropriation, which was reflected in the abductive appropriation framework we proposed here. Appropriation stages are built on top of interpretation states that enable users to organize rich interactive discourses with the technology. Users interpretations do not refer only to what designers mean with the technology but also to what the technology has come to mean to the users. Thus, appropriation is tied to the practical uses the users can make of the technology in their lives based on their understanding of it.

Appropriation evolves as interpretation evolves and communicative breakdowns sign remarkable moments where users' interpretations are exposed, usually during a process of review or refinement. As in standard social communication processes, skillful interlocutors would monitor and pay special attention to these moments in order to assure that their intents are communicated effectively, efficiently, and "appropriately." In the terms of Semiotic Engineering, efficient and effective interactive communication is achieved by means of good communicability. The semiotic qualities we suggest here are targeted at improving IoT technology communicability to support users' interpretations and abductive reasoning.

Through breakdowns, users communicate, even if in a rudimentary and unarticulated way, their very own interpretation of the technology. The communicability evaluation tags help us understand what the users were saying. Our findings suggest that they have the potential to systematically point at the directions of how IoT technology appropriation will unfold, leading to successful or flawed adoption. These patterns of failures in the designer-to-user communication, across devices and across interfaces, as they accumulate, hold the potential to help us see powerful design principles in operation that can determine successful appropriation of IoT technology by users. Our semiotic account is a step towards reveling and understanding these design principles.

\section{ACKNOWLEDGMENT}

We thank all the anonymous reviewers for their valuable feedback and suggestions. We thank Professor Alessandro Garcia from PUC-Rio for the support without which this research would not be possible. We thank Professor Dr. Volkmar Pipek from the University of Siegen for the collaboration and insightful discussions. We thank our colleagues from our research groups SERG at PUC-Rio and CRADL at UCI for thoughtful discussions and feedback. Most of all, we thank all volunteers for their participation in the study. Finally, Bruno A. Chagas thanks CAPES (Grant No. 88887-122734/2016-00) and CNPq (Grants No. 153902/2015-9 and 140568/2018-2) and Clarisse S. de Souza thanks CNPq (Grant 304224/2017-0) for partially funding this research.

\section{REFERENCES}

[1] B. A. Chagas, D. F. Redmiles, and C. S. de Souza, "Observed Appropriation of IoT Technology: A Semiotic Account," in Proceedings of the 17th Brazilian Symposium on Human Factors in Computing Systems, New York, NY, USA, 2018, pp. 33:1-33:10.

[2] J. Gaskin and K. Lyytinen, "Psychological Ownership and the Individual Appropriation of Technology," in Information Systems Theory, Springer, New York, NY, 2012, pp. 25-39.

[3] E. M. Rogers, Diffusion of Innovations, 5th Edition, 5th edition. New York: Free Press, 2003.

[4] C. Humphreys-Jones, "An investigation of the types and structure of misunderstandings," Thesis, Newcastle University, 1986.

[5] C. Bazzanella and R. Damiano, "The interactional handling of misunderstanding in everyday conversations," J. Pragmat., vol. 31, no. 6, pp. 817-836, Jun. 1999.

[6] B. Hansen, D. Novick, and S. Sutton, "Prevention and repair of breakdowns in a simple task domain," in Proceedings of the AAAI-96 Workshop on Detecting, Repairing, and Preventing Human-Machine Miscommunication, 1996, pp. 5-12.

[7] T. Winograd and F. Flores, Understanding Computers and Cognition: A New Foundation for Design. Intellect Books, 1986.

[8] K. Ashton, "That 'internet of things' thing," RFiD J., vol. 22, no. 7, pp. 97-114, 2009.

[9] S. K. Anithaa et al., "The Internet of Things-A survey," World Sci. News, vol. 41, p. 150, 2016.

[10] L. Atzori, A. Iera, and G. Morabito, "The internet of things: A survey," Comput. Netw., vol. 54, no. 15, pp. 2787-2805, 2010.

[11] J. Gubbi, R. Buyya, S. Marusic, and M. Palaniswami, "Internet of Things (IoT): A vision, architectural elements, and future directions," Future Gener. Comput. Syst., vol. 29, no. 7, pp. 1645-1660, Sep. 2013.

[12] H. Kopetz, "Internet of Things," in Real-Time Systems, Springer, Boston, MA, 2011, pp. 307-323.

[13] I. Lee and K. Lee, "The Internet of Things (IoT): Applications, investments, and challenges for enterprises," Bus. Horiz., vol. 58, no. 4, pp. 431-440, Jul. 2015.

[14] S. Li, L. D. Xu, and S. Zhao, "The internet of things: a survey," Inf. Syst. Front., vol. 17, no. 2, pp. 243-259, Apr. 2015.

[15] F. Xia, L. T. Yang, L. Wang, and A. Vinel, "Internet of Things," Int. J. Commun. Syst., vol. 25, no. 9, pp. 1101-1102, Sep. 2012.

[16] B. L. Risteska Stojkoska and K. V. Trivodaliev, "A review of Internet of Things for smart home: Challenges and solutions," J. Clean. Prod., vol. 140, pp. 1454-1464, Jan. 2017.

[17] A. Zanella, N. Bui, A. Castellani, L. Vangelista, and M. Zorzi, "Internet of Things for Smart Cities," IEEE Internet Things J., vol. 1, no. 1, pp. 22-32, Feb. 2014.

[18] L. D. Xu, W. He, and S. Li, "Internet of Things in Industries: A Survey," IEEE Trans. Ind. Inform., vol. 10, no. 4, pp. 2233-2243, Nov. 2014.

[19] M. Maksimović, V. Vujović, and B. Periśić, "A custom Internet of Things healthcare system," in 2015 10th Iberian Conference on Information Systems and Technologies (CISTI), 2015, pp. 1-6. 
[20] G. Kortuem, F. Kawsar, V. Sundramoorthy, and D. Fitton, "Smart objects as building blocks for the Internet of things," IEEE Internet Comput., vol. 14, no. 1, pp. 44-51, Jan. 2010.

[21] P. Dourish, "The Appropriation of Interactive Technologies: Some Lessons from Placeless Documents," Comput. Support. Coop. Work CSCW, vol. 12, no. 4, pp. 465-490, Dec. 2003.

[22] S. Bødker and E. Christiansen, "Poetry in Motion: Appropriation of the World of Apps," in Proceedings of the 30th European Conference on Cognitive Ergonomics, New York, NY, USA, 2012, pp. 78-84.

[23] C. T. de Campos, "O processo de apropriação do desenho à escrita," Dissertação de Mestrado, Universidade Federal de São Carlos, São Carlos, SP, Brasil, 2011.

[24] M. Magnusson and N. Pramling, "Signs of knowledge: the appropriation of a symbolic skill in a five-year-old," Eur. Early Child. Educ. Res. J., vol. 19, no. 3, pp. 357-372, Sep. 2011.

[25] B. Rogoff, "Observing sociocultural activity on three planes: Participatory appropriation, guided participation, and apprenticeship," Pedagogy Pract. Cult. Identities, pp. 58-74, 2008.

[26] J. F. de F. Fernandes, M. G. Carvalho, and E. N. Campos, "Vygotsky and Bakhtin: the educational action as a dialogic project of meaning production," Bakhtiniana Rev. Estud. Discurso, vol. 7, no. 2, pp. 95108, Dec. 2012

[27] A. Dix, "Designing for Appropriation," in Proceedings of the 21st British HCI Group Annual Conference on People and Computers: HCI...But Not As We Know It - Volume 2, Swindon, UK, 2007, pp. $27-$ 30.

[28] J. P. Bansler and E. Havn, "Sensemaking in Technology-Use Mediation: Adapting Groupware Technology in Organizations," Comput. Support. Coop. Work CSCW, vol. 15, no. 1, pp. 55-91, Feb. 2006.

[29] S. Draxler and G. Stevens, "Supporting the Collaborative Appropriation of an Open Software Ecosystem," Comput. Support. Coop. Work CSCW, vol. 20, no. 4-5, pp. 403-448, Oct. 2011.

[30] J. Carroll, S. Howard, J. Peck, and J. Murphy, "From Adoption to Use: the process of appropriating a mobile phone," Australas. J. Inf. Syst., vol. 10 , no. $2,2003$.

[31] G. O. Wiredu, "User appropriation of mobile technologies: Motives, conditions and design properties," Inf. Organ., vol. 17, no. 2, pp. 110 129, Jan. 2007.

[32] W. Wirth, T. V. Pape, and V. Karnowski, "An Integrative Model of Mobile Phone Appropriation," J. Comput.-Mediat. Commun., vol. 13, no. 3, pp. 593-617, Apr. 2008.

[33] T. Jakobi, C. Ogonowski, N. Castelli, G. Stevens, and V. Wulf, "The Catch(Es) with Smart Home: Experiences of a Living Lab Field Study," in Proceedings of the 2017 CHI Conference on Human Factors in Computing Systems, New York, NY, USA, 2017, pp. 1620-1633.

[34] T. Ludwig, A. Boden, and V. Pipek, "3D Printers As Sociable Technologies: Taking Appropriation Infrastructures to the Internet of Things," ACM Trans Comput-Hum Interact, vol. 24, no. 2, pp. 17:117:28, Apr. 2017.

[35] J. Carroll, "Completing design in use: closing the appropriation cycle," ECIS 2004 Proc., p. 44, 2004

[36] G. Stevens, V. Pipek, and V. Wulf, "Appropriation Infrastructure: Supporting the Design of Usages," in End-User Development, 2009, pp. 50-69.

[37] J. Carroll, S. Howard, F. Vetere, J. Peck, and J. Murphy, "Just what do the youth of today want? Technology appropriation by young people," in Proceedings of the 35th Annual Hawaii International Conference on System Sciences, 2002, pp. 1777-1785.

[38] E. Karapanos, J. Zimmerman, J. Forlizzi, and J.-B. Martens, "User Experience over Time: An Initial Framework," in Proceedings of the SIGCHI Conference on Human Factors in Computing Systems, New York, NY, USA, 2009, pp. 729-738.

[39] C. S. De Souza, The semiotic engineering of human-computer interaction. Cambridge, Massachusetts: MIT press, 2005.

[40] J. Hoopes, Ed., Peirce on Signs: Writings on Semiotic by Charles Sanders Peirce, New edition edition. Chapel Hill: The University of North Carolina Press, 1991

[41] H. Meadan, M. M. Ostrosky, and J. W. Halle, “'What?'; 'I Don't Understand'; and 'Pardon?': Using Communication Breakdowns to Encourage Communication," Young Except. Child., vol. 9, no. 3, pp. 2-9, Apr. 2006.
[42] S. P. Urquijo, S. A. R. Scrivener, and H. K. Palmén, "The Use of Breakdown Analysis in Synchronous CSCW System Design," in Proceedings of the Third European Conference on ComputerSupported Cooperative Work 13-17 September 1993, Milan, Italy ECSCW '93, G. de Michelis, C. Simone, and K. Schmidt, Eds. Springer Netherlands, 1993, pp. 281-293.

[43] M. Sharples, "A study of breakdowns and repairs in a computermediated communication system," Interact. Comput., vol. 5, no. 1, pp. 61-77, Mar. 1993

[44] V. Pipek and V. Wulf, "Infrastructuring: Toward an integrated perspective on the design and use of information technology," J. Assoc. Inf. Syst., vol. 10, no. 5, p. 447, 2009.

[45] R. O. Prates, C. S. de Souza, and S. D. J. Barbosa, "Methods and Tools: A Method for Evaluating the Communicability of User Interfaces," interactions, vol. 7, no. 1, pp. 31-38, Jan. 2000.

[46] C. S. De Souza and C. F. Leitão, Semiotic engineering methods for scientific research in HCI. Morgan \& Claypool Publishers, 2009.

[47] C. S. De Souza, R. O. Prates, and S. D. J. Barbosa, "A Method for Evaluating Software Communicability," in Proceedings of the Second Brazilian Workshop in Human-Computer Interaction, Campinas, SP, Brazil, 1999.

[48] E. Goodman, M. Kuniavsky, and A. Moed, Observing the User Experience, Second Edition: A Practitioner's Guide to User Research, 2nd edition. Morgan Kaufmann, 2012.

[49] V. Braun and V. Clarke, "Using thematic analysis in psychology," Qual. Res. Psychol., vol. 3, no. 2, pp. 77-101, Jan. 2006.

[50] L. Marques Afonso, "Communicative Dimensions of Application Programming Interfaces (APIs)," DOUTOR EM CIÊNCIAS INFORMÁTICA, PONTIFÍCIA UNIVERSIDADE CATÓLICA DO RIO DE JANEIRO, Rio de Janeiro, Brazil, 2015.

[51] C. S. De Souza, R. O. Prates, and T. Carey, "Missing and declining affordances: are these appropriate concepts?," J. Braz. Comput. Soc., vol. 7, no. 1, pp. 26-34, 2000.

[52] J. Carroll, S. Howard, F. Vetere, J. Peck, and J. Murphy, "Identity, power and fragmentation in cyberspace: technology appropriation by young people," in Proceedings of the Twelfth Australasian Conference on Information Systems, 2001, p. 6.

[53] H. Lieberman, F. Paternò, M. Klann, and V. Wulf, "End-user development: An emerging paradigm," in End user development, Springer, 2006, pp. 1-8.

[54] B. A. Chagas, D. F. Redmiles, and Clarisse. S. De Souza, "End-user development for the Internet of Things OR How can a (smart) light bulb be so complicated?," in 2017 IEEE Symposium on Visual Languages and Human-Centric Computing (VL/HCC), 2017, pp. 273277.

[55] B. A. Chagas, H. Fuks, and C. S. de Souza, "Lessons Learned in the Design of Configurable Assistive Technology with Smart Devices," in Fifth International Symposium on End User Development, 2015, vol. 9083, pp. 180-185.

[56] D-Link, "DCH-S150 mydlink Wi-Fi Motion Sensor." [Online]. Available: http://www.dlink.co.in/products/?pid=697. [Accessed: 12Jan-2019].

[57] R. de A. Maués and S. D. J. Barbosa, "Cross-Communicability: Evaluating the Meta-communication of Cross-Platform Applications," in Human-Computer Interaction - INTERACT 2013, Springer, Berlin, Heidelberg, 2013, pp. 241-258.

[58] R. de A. Maués and S. D. J. Barbosa, "Reflections on the CrossPlatform Semiotic Inspection Method," in Human-Computer Interaction. Theories, Methods, and Tools, Springer, Cham, 2014, pp. 533-544.

[59] A. Kuper, L. Lingard, and W. Levinson, "Critically appraising qualitative research," BMJ, vol. 337, p. a1035, Aug. 2008.

[60] C. W. Morris, "Foundations of the Theory of Signs," in International Encyclopedia of Unified Science, vol. I, No. 2., Chicago University Press, 1938, pp. 1-59.

[61] L. Horn and G. Ward, The Handbook of Pragmatics. John Wiley \& Sons, 2008

[62] K. Doherty and G. Doherty, "The construal of experience in HCI: Understanding self-reports,” Int. J. Hum.-Comput. Stud., vol. 110, pp. 63-74, Feb. 2018. 